\title{
Proof of a Multipole Conjecture due to Geroch
}

\author{
R. Beig and W. Simon \\ Institut für Theoretische Physik, Universität Wien, A-1090 Vienna, Austria
}

\begin{abstract}
A result, first conjectured by Geroch, is proved to the extent, that the multipole moments of a static space-time characterize this space-time uniquely. As an offshoot of the proof one obtains an essentially coordinate-free algorithm for explicitly writing down a geometry in terms of it's moments in a purely algebraic manner. This algorithm seems suited for symbolic manipulation on a computer.
\end{abstract}

\section{Introduction}

In the literature on General Relativity there have been several approaches to multipole moments in the static or more general context, e.g. the one of Clarke and Sciama [1], based on a certain eigenvalue problem. Another one, due to Geroch $[2,3]$, which uses a conformal compactification of 3-space, has found special attention because of the elegant geometric way in which the origin-dependence of multipole moments can be expressed. Our work is based on Geroch's approach.

Multipole moments in General Relativity provide, or should provide, an invariant way of interpreting exact or approximate solutions to Einstein's equations. Of course, this is true only if such solutions are uniquely determined by these moments up to isometries. The latter statement, which is the content of Geroch's "Conjecture 1" [3], is proved in the present paper. Partial results on this question have before been obtained by Xanthopoulos [4].

The strategy of our proof parallels the corresponding Newtonian proof. There one proceeds in two steps: In Step 1 one shows that the so-called Kelvin transform, which is the flat-space analogue of Geroch's compactification trick leaves the field equation, namely Laplace's equation, invariant. Therefore the potential, if it goes to zero for large distances from the source, is analytic near the origin $\Lambda$ of the compactified space. In Sect. 3 we prove an analogous result within Einstein's theory. In the finite region the analyticity of the field variables is, in fact, well known [5]. The situation is considerably trickier at infinity, due to the fact that Einstein's equations, far from being conformally invariant, become formally singular at the point $\Lambda$. (One faces a similar problem in the characteristic initial value problem at null infinity [6].) The essential idea to overcome this 
difficulty consists in introducing the formally singular terms as additional field variables and deriving regular differential equations for them in such a way that the whole set of variables satisfies an elliptic system. This implies analyticity. It is worth pointing out that all this can be done for $n=3$-dimensional spaces only (where the Weyl tensor vanishes identically). Quite contrary to Newtonian theory, neither the analyticity theorem nor Conjecture 1 itself can be expected to hold in general if $n>3$.

Step 2 in the Newtonian proof consists in simply noting that an analytic function is determined by it's value and the value of it's derivatives at a point. The value of the suitably rescaled ("unphysical") Newtonian potential and the trace-free parts of it's derivatives at $\Lambda$ are, by definition, the multipole moments. The trace parts have to vanish by virtue of Laplace's equation. In Sect. 4 we give the general relativistic counterpart to Step 2. One of the new features is, that the trace-parts of the derivatives at $\Lambda$ of the unphysical quantities are nonzero and, in any multipole order, have to be determined from the lower orders. As expected, "orders" in this scheme correspond essentially to order in a $1 / r$-expansion of the physical variables.

\section{The Assumptions}

We consider a smooth 3-manifold $\tilde{X}$ endowed with a smooth, positive definite metric $\tilde{g}_{i j}$. We shall write: $D_{i}$ for covariant differentiation; $\Delta$ for the covariant Laplace operator. $D_{[i} D_{j]} V_{k}=\frac{1}{2} R_{i j k}{ }^{h} V_{h}, R_{i j}=R^{k}{ }_{i k j}$ and $R:=R_{i}^{i}$ define the Riemann tensor and its contractions. The tildes distinguish the variables in the "physical" space $\tilde{X}$ from those living in the compactification $X$. On $\tilde{X}$, we are also given a smooth scalar field $\tilde{U}$, such that

$$
\begin{aligned}
\tilde{\Delta} \tilde{U} & =0 \\
\tilde{R}_{i j} & =2 \tilde{D}_{i} \tilde{U} \tilde{D} \tilde{U}_{j} .
\end{aligned}
$$

These equations are equivalent to Einstein's static field equations in vacuo for the metric

$$
d s^{2}=e^{2 \tilde{U}} d t^{2}-e^{-2 \tilde{U}} \tilde{g}_{i j} d x^{i} d x^{j}
$$

We now demand that $\left(\tilde{X}, \tilde{U}, \tilde{g}_{i j}\right)$ be asymptotically flat and of non-vanishing mass $m$. This is equivalent to the following set of assumptions. First of all, there should exist a manifold $X$, consisting of $\tilde{X}$ plus one additional point $\Lambda$. Moreover there exists a constant $m \neq 0$, such that the function $\omega=m^{-2} \tilde{U}^{2}$ is $C^{2}$ on $X$ and satisfies

$\left.B_{1}\right)\left.\omega\right|_{\Lambda}=0$ and $\left.D_{i} \omega\right|_{\Lambda}=0$.

$\left.B_{2}\right) g_{i j}=\omega^{2} \tilde{g}_{i j}$ extends to a $C^{4, \alpha}$-metric on $X$. (In particular, $\omega \neq 0$ outside $\Lambda$.)

(A function is said to be of class $C^{k, \alpha}$, iff it's $k$-th derivatives exist and are

Hölder-continuous with exponent $0<\alpha<1$.) Furthermore

$$
\left.D_{i} D_{j} \omega\right|_{\Lambda}=2 \lambda_{i j}, \quad \text { where } \lambda_{i j}:=\left.g_{i j}\right|_{\Lambda} .
$$

The sign of $m$ is fixed by $\tilde{U}=-m|m| \omega^{1 / 2}$. 
Remark. We have found it convenient to state our requirements and work always in terms of a particular conformal factor. In the appendix we make contact with conditions á la Geroch [3], where one assumes just the existence of some conformal factor satisfying $\left.B_{1}\right), B_{2}$ ).

Furthermore, by extending the results in [7] and [8], it can be shown [9] that $\left.B_{1}\right), B_{2}$ ) hold for static solutions which are asymptotically Euclidean in the standard coordinate-dependent sense and of nonvanishing (Komar-) mass.

In Sect. 4 there will arise a potential difference to Geroch's work. Namely, along with each choice of potential ( $\tilde{\psi}$ in Geroch's case, $\tilde{U}$ in our case) there goes a particular definition of multipole moments which could, in principle, be different. Consider, e.g. the one-parameter family of potentials $\tilde{U}_{a}=$ $\frac{1}{2 a} \sinh 2 a \tilde{U}(a \in \mathbb{R})$. Our $\tilde{U}$ is equal to $\tilde{U}_{0}$. Geroch's $\tilde{\psi}$ corresponds to $\tilde{U}_{1 / 4}$ and $\tilde{U}_{1}, \tilde{U}_{1 / 2}$ are the static limits of the potentials chosen by Hansen [10] and Hoenselaers [11], respectively. More generally, let $f: \mathbb{R} \rightarrow \mathbb{R}$ be any analytic function satisfying $f(x)=-f(-x)$ and $\frac{d f}{d x}(0)=1$ and define a new potential $\tilde{V}$ by $\tilde{V}=f(\tilde{U})$. Then, using the methods of the following two paragraphs, it should be possible to show that $\tilde{U}$ and $\tilde{V}$ produce the same set of multipole moments for a given solution.

As to terminology, we finally remark that we shall sometimes refer to a solution $\left(\tilde{U}, \tilde{g}_{i j}\right)$ of $(1,2)$ on $\tilde{X}$ which satisfies $\left.\left.B_{1}\right), B_{2}\right)$ as "a solution". Two solutions will be said to be equal iff the $\tilde{U}$ 's in both cases agree and the $\tilde{g}_{i j}$ 's are isometric to each other (these conditions being equivalent, of course, to the fact that the 4-manifolds in both cases are isometric).

\section{The Analyticity Theorem}

Theorem 1. For any solution $\left(\tilde{U}, \tilde{g}_{i j}\right)$ there exists in $X$ a chart defined in some neighbourhood of $\Lambda$, such that $g_{i j}$, $\omega$ are both analytic.

Proof. Using standard formulas for conformal transformations, the field equations (1), (2) lead to

$$
\begin{gathered}
\Delta \omega=3 m^{-2} R \\
\omega R_{i j}=\frac{1}{2} m^{2} D_{i} \omega D_{j} \omega-D_{i} D_{j} \omega+\frac{1}{3} g_{i j} \Delta \omega .
\end{gathered}
$$

By virtue of $B_{2}$ ), (4) is elliptic with $C^{2, \alpha}$-coefficients. Hence $\omega$ is $C^{4, \alpha}$ on general grounds [12]. Operating on the contracted version of (5) with $D_{i}$, using (4) and, once more, (5) gives

$$
D_{i} R=m^{2} R D_{i} \omega-m^{2} R_{i j} D^{j} \omega .
$$

Next we operate on (5) with $D_{k}$ and antisymmetrize with respect to $i$ and $k$. Using (6) we arrive at

$$
\omega\left(D_{[k} R_{i] j}-\frac{1}{2} m^{2} D_{[k} \omega R_{i] j}\right)=0 .
$$


The bracket vanishes outside $\Lambda$ and, by continuity, also at $\Lambda$. Operating on this expression with $D^{k}$ once more, commuting derivatives in $D_{k} D_{i} R_{k j}$ and using

$$
R_{h i j k}=2\left(g_{h[j} R_{k] i}-g_{i[j} R_{k] h}\right)-R g_{h[j} g_{k] i}
$$

for the Riemann tensor in 3 dimensions, we find with the help of (4)-(7)

$$
\begin{aligned}
\Delta R_{i j}= & \frac{1}{2} m^{4} R D_{i} \omega D_{j} \omega-m^{4} D^{k} \omega R_{k(i} D_{j)} \omega+m^{2} \omega R_{i k} R_{j}^{k}+3 R_{i k} R_{j}^{k}-g_{i j} R_{k l} R^{k l} \\
& -R R_{i j}+g_{i j} R^{2} .
\end{aligned}
$$

Now observe that the quantity

$$
\sigma_{i j}:=\omega^{-1}\left(\frac{1}{2} m^{2} D_{i} \omega D_{j} \omega-D_{i} D_{j} \omega+\frac{1}{3} g_{i j} \Delta \omega\right),
$$

though undefined at $A$, can be extended to be $C^{2, \alpha}$ there by virtue of (5) since $R_{i j}$ is $C^{2, \alpha}$. Because of (4) and (8), our static solutions satisfy the following system of partial differential equations in the variables $\omega, g_{i j}$ and $\sigma_{i j}\left(\sigma:=\sigma_{i}^{i}\right)$

$$
\begin{gathered}
\Delta \omega=3 m^{-2} \sigma \\
R_{i j}\left(g_{k l}\right)=\sigma_{i j} \\
\Delta \sigma_{i j}=\frac{1}{2} m^{4} \sigma D_{i} \omega D_{j} \omega-\ldots+g_{i j} \sigma^{2} .
\end{gathered}
$$

Next we transform to harmonic coordinates which satisfy

$$
\Delta x^{\prime} i=0
$$

with $\left|\partial x^{i} / \partial x^{j}\right|$ nonvanishing at $\Lambda$. Such coordinates can always be found in some neighbourhood of $\Lambda$ [13]. Since the coefficients of the elliptic equation (13) are $C^{3, \alpha}$, the $x^{i}\left(x^{j}\right)$ will, on general grounds [12], be at least $C^{5, \alpha}$. Therefore $g_{i j}^{\prime}$ is still $C^{4, \alpha}, \sigma_{i j}^{\prime}$ is $C^{2, \alpha}$ and $\omega^{\prime}$ is $C^{4, \alpha}$. Omitting the primes, we have a $C^{2, \alpha}$-solution $\left(\omega, g_{i j}, \sigma_{i j}\right)$ to $(10),(11)$ and (12) which, in these coordinates, form an elliptic system. (Due to the appearance of second derivatives of $g_{i j}$ in (12), it is not strongly elliptic; see [14] for the definitions.) Hence, from a theorem of Morrey [14], $\omega, g_{i j}$ and $\sigma_{i j}$ are all analytic near $\Lambda$. This proves Theorem 1 .

\section{The Multipole Theorem}

Following the scheme given by Geroch in [3] we define recursively a set $P, P_{i}$, $P_{i j}, \ldots$ to totally symmetric, trace-free tensor fields by

$$
\begin{gathered}
P=-m \\
P_{i_{1} \ldots i_{s+1}}=\rho\left[D_{i_{1}} P_{i_{2} \ldots i_{s+1}}-\frac{s}{2}(2 s-1) R_{i_{1} i_{2}} P_{i_{3} \ldots i_{s+1}}\right]
\end{gathered}
$$

where $\rho\left[T_{i \ldots j}\right]$ denotes the totally symmetric, trace-free part of $T_{i \ldots j}$. The $2^{s}$ moment $M_{i_{1} \ldots i_{s}}$ is defined to be the value of $P_{i_{1} \ldots i_{s}}$ at $\Lambda$. (Since $P$ is constant, $M_{i}$ vanishes so that we are automatically in the "centre of mass".) This construction can be considered as mapping every solution $\left(\tilde{U}, \tilde{g}_{i j}\right)$ into the collection of tensors $\left(\lambda_{i j}, M, M_{i}=0, M_{i_{1}, i_{2}, \ldots}\right)$. Among the set of all these collections, identify the 
elements which are connected by some element of $G L(3, \mathbb{R})$. There results a set $E$ of equivalence classes $E=\{e, f, \ldots\}$.

Theorem 2. The map $\left(\tilde{U}, \tilde{g}_{i j}\right) \rightarrow e$ is an injective function, that is to say, if two solutions lead to the same equivalence class $e$, they are identical at least in some neighbourhood of $\Lambda$.

Proof. The idea is to reconstruct $\left(\tilde{U}, \tilde{g}_{i j}\right)$ from $e$ in the following way: We compute $\omega, R_{i j}$ and their covariant derivatives at $\Lambda$. It then follows from a classical theorem, a modern version of which can be found in [15], that $g_{i j}$ is determined. Hence $\tilde{g}_{i j}=\omega^{-2} g_{i j}$ and $\tilde{U}=-m|m| \omega^{1 / 2}$ are unique.

In order to start an induction process, observe that, by assumption $B_{2}$ and (4), $\left.R\right|_{A}=2 M^{2}$. Using this equation and (14), we have

$$
\left.R_{i j}\right|_{\Lambda}=-2 M^{-1} M_{i j}+\frac{2}{3} M^{2} \lambda_{i j} .
$$

Let us now assume that after $n-1$ steps we know $R_{i j}$ (and hence $R_{h i j k}$ ) and its covariant derivatives up to order $n-1$ and $\omega$ and its derivatives up to order $n+1$. Then, taking $D_{i_{1} \ldots} D_{i_{n-1}} D^{j}$ of (5) and commuting derivatives, if necessary, by use of the Ricci identity, we obtain $D_{i_{1}} \ldots D_{i_{n-1}} D_{i} \Delta \omega$ expressed in terms of known quantities. (Since $\left.\omega\right|_{\Lambda}=0$, the $n$-th derivative of $R_{i j}$ does not contribute). With the help of (4) we have the value of $D_{i_{1}} \ldots D_{i_{n}} R$ at $\Lambda$. Substituting back into the $n$-th derivatives of (5), we obtain all the $n+2$-derivatives of $\omega$. From the definition (14) we see that

$$
-\frac{M}{2} \rho\left(D_{i_{1}} \ldots D_{i_{n}} R_{i_{n+1} i_{n+2}}\right)=P_{i_{1} \ldots i_{n+2}}+\text { known terms. }
$$

$D_{\left(i_{1} \ldots\right.} D_{i_{n}} R_{i_{n+1} i_{n+2)}}$ is known up to trace terms of which there are three types:

a) $D_{i_{1} \ldots i_{n}} R$, which has been just computed.

b) $D_{i_{1}} \ldots D^{j} \ldots D_{i_{n-1}} R_{i_{n} j}$ can be reduced to a) by virtue of Ricci and Bianchi identities.

c) $D_{i_{1}} \ldots \Delta \ldots D_{i_{n-2}} R_{i_{n-1} i_{n}}$ can be determined from known quantities by help of the $n-2$-th derivative of Eqn. (8).

$D_{i_{1}} \ldots D_{i_{n}} R_{i_{n+1} i_{n+2}}$ can be written as $D_{\left(i_{1}\right.} \ldots D_{i_{n}} R_{\left.i_{n+1} i_{n+2}\right)}$ plus terms involving antisymmetrization with respect to at least one pair of indices. If both indices are in the derivatives, we can again use the Ricci identity to reduce them to known terms. If one index occurs in the Ricci tensor, we can appeal to the $n-1$-th derivative of (7) (with $\omega$ divided out). This finishes the proof.

We finally remark that one knows the explicit expressions for the Taylor coefficients in normal coordinates of an analytic metric in terms of the curvature and it's derivatives [16], whereas for the scalar $\omega$ we simply have

$$
\omega=\lambda_{i j} x^{i} x^{j}+\left.\sum_{r=3}^{\infty} \frac{1}{r !} D_{i_{1}} \ldots D_{i_{r}} \omega\right|_{\Lambda} x^{i_{1}} \ldots x^{i_{r}}
$$

in normal coordinates $x^{i}$ centered at $\Lambda$.

The reduction procedure above seems suited for algebraic computer manipulation. If one was lucky, one might even find general recursion formulas for $\left(\Omega, g_{i j}\right)$ or $\left(\tilde{U}, \tilde{g}_{i j}\right)$ in terms of the moments. 


\section{Remaining Problems}

We have not touched so far Geroch's 2nd Conjecture [3]. In the present context it should mean that the equations for the "unphysical" variables $\omega, g_{i j}$ can be formally satisfied to all orders by the above inductive scheme without further restrictions on the multipole moments. Although it is quite suggestive that this is the case, a formal proof seems to be a nontrivial algebraic problem. One has to show that the quantities $D_{i_{1} \ldots i_{n}} R_{i_{n+1} i_{n+2}}$ which are computed in the proof of Theorem 2, satisfy the consistency conditions such that they

a) belong to a metric which, together with $\omega$,

b) satisfies all the equations. (In this connection, compare [17].) On top of that,

c) one must find suitable fall-off-conditions for large order on the moments such that the power series' in terms of which the formal solution is written down have nonvanishing radius of convergence.

If these problems are solved (partial results, using a different approach, can be found in [9]), the "physical" space with the metric $\tilde{U}:=-m|m| \omega^{1 / 2}$ and $\tilde{g}_{i j}:=\omega^{-2} g_{i j}$ can be constructed $\left(\omega\right.$ is positive near $\Lambda$ because of $\left.\left.D_{i} D_{j} \omega\right|_{A}=2 \lambda_{i j}\right)$.

Another problem is to find the extension of our considerations to stationary space-times with or without additional zero-rest-mass fields $[10,11]$. There arises the question of specifying the conformal factor $\Omega$ in order to get an elliptic system similar to (10), (11) and (12) for $\Omega, g_{i j}, R_{i j}$ and some suitably defined potentials.

\section{Appendix}

We intend to outline the relationship between our assumptions $B_{1}$ ), $B_{2}$ ) and requirements which are more in the spirit of Geroch's original paper [3]. We assume that there exists on $X$ a $C^{2}$-function $\Omega$ such that $\left.A_{1}\right)\left.\Omega\right|_{\Lambda}=0,\left.D_{i} \Omega\right|_{\Lambda}=0$.

$\left.A_{2}\right) g_{i j}=\Omega^{2} \tilde{g}_{i j}$ is a $C^{4, \alpha}$-metric on $X$ and $\left.\left(D_{i} D_{j} \Omega-2 g_{i j}\right)\right|_{\Lambda}=0$.

$\left.A_{3}\right) \Omega^{-2} \tilde{R}$ is $C^{2}$ on $X$ and nonzero at $\Lambda$. Finally,

$\left.A_{4}\right) \tilde{U} \rightarrow 0$ as the argument approaches $\Lambda$.

We first show that $\left.\left.B_{1}\right), B_{2}\right) \rightarrow A_{1}$ ) to $A_{4}$ ). Setting $\Omega=\omega, A_{1}$ ) and $A_{2}$ ) are satisfied. To prove $A_{3}$ ), note that $\tilde{R} / \omega^{2}=R=\sigma$, which, from $B_{2}$ ), is $C^{2}$. Moreover, $\left.\left.\sigma\right|_{A}=2 m^{2} \neq 0, A_{4}\right)$ is immediate from $\omega=m^{-2} \tilde{U}^{2}$.

To go the opposite way, we first show that $U=\Omega^{-1 / 2} \tilde{U}$ is $C^{4, \alpha}$ in $X$. Writing $\Omega^{-2} \widetilde{R}=\kappa^{4}$, we have, by specializing (2.20) of Hansen [10],

$$
\left(\Delta-\frac{R}{8}\right) \kappa=\frac{3}{8} \kappa^{5}+\kappa^{-7} C_{i j k} C^{i j k}
$$

where $C_{i j k}$ is the Bach tensor. From (15) we see that $\kappa$ is $C^{3, \alpha}$ (since $C_{i j k}$ contains third derivatives of $g_{i j}$ ). Furthermore, from (1), $U$ satisfies

$$
\left(\Delta-\frac{R}{8}\right) U=-\frac{\kappa^{4}}{8} U \text { in } \quad X \backslash\{\Lambda\} .
$$


Now draw a sphere $\Sigma$ enclosing the point $\Lambda$ and seek a $C^{4, \alpha}$-solution $W$ of $(16) \ln X$ with $U=W$ on $\Sigma$. For sufficiently small $\Sigma$, this $W$ will always exist. Defining $\tilde{Z}=\tilde{U}-\Omega^{1 / 2} W$ and going back to the physical space $\tilde{X}$, we have

$$
\tilde{\Delta} \tilde{Z}=0 \text {. }
$$

Now observe that $\tilde{Z}$ vanishes on $\Sigma$ and, by $A_{4}$ ), also at $\Lambda$. Hence the maximum principle implies that $\tilde{Z}=0$ in $\tilde{X}$. Therefore $U$ can be extended to a $C^{4, \alpha}$-solution of (16) by setting $\left.U\right|_{\Lambda}=\left.W\right|_{\Lambda}$.

From (2) we have

$$
\Omega^{-2} \tilde{R}=\frac{1}{2} \Omega^{-1} U^{2} D^{i} \Omega D_{i} \Omega+2 U D^{i} \Omega D_{i} U+2 \Omega D^{i} U D_{i} U .
$$

Hence $\left.\Omega^{-2} \tilde{R}\right|_{A}=\left.2 U^{2}\right|_{A} \neq 0$, using l'Hospital's rule. Setting $m=-\left.U\right|_{A}$, it can now be seen that $\omega=m^{-2} \widetilde{U}^{2}$ satisfies $B_{1}$ ), $B_{2}$ ), at least in some neighbourhood of $\Lambda$, which is all we need for our purposes.

As a final remark we note that Geroch's potential $\psi(\tilde{\psi}=2 \sinh \tilde{U} / 2)$ which satisfies the conformally invariant equation

$$
\left(\Delta-\frac{R}{8}\right) \psi=0
$$

can be shown to be $C^{4, \alpha}$ without requiring $\kappa$ to be $C^{2}$ in $A_{3}$ ). So we could have used $\Omega=M^{-2} \tilde{\psi}^{2}$ in the whole of this work instead of $\Omega=\omega$. It turns out, however, that $A_{3}$ ) then has to be used at some other place in the proof of the analogue of Theorem 1. Furthermore the equations become more complicated. So we chose to work in terms of $\Omega=\omega$.

Acknowledgements. One of us (R.B.) thanks B. Schmidt for discussions in the prenatal stages of this work. Several helpful conversations with $\mathrm{H}$. Urbantke are also gratefully acknowledged.

\section{References}

1. Clarke, C. Sciama, D. : Static gravitational multipoles. The connection between field and sources in general relativity. Gen. Rel. Grav. 2, 331 (1971)

2. Geroch, R. : Multipole moments. I flat space. J. Math. Phys. 11, 1955 (1970)

3. Geroch, R. : Multipole moments. II curved space. J. Math. Phys. 11, 2580 (1970)

4. Xanthopoulos, B. C. : Multipole moments in general relativity. J. Phys. A. 12, 1025 (1979)

5. Müller zum Hagen, H.: On the analyticity of stationary vacuum solutions of Einstein's equations. Proc. Cambridge Philos. Soc. 68, 199 (1970)

6. Friedrich, H. : On the regular and the asymptotic characteristic initial value problem for Einstein's vacuum field equations. Proceedings of the 3rd Gregynog relativity workshop (ed. M. Walker), MPI preprint (1979)

7. Beig, R. : The static gravitational field near spatial infinity, I. Gen. Rel. Grav. 12, 439 (1980)

8. Beig, R., Simon, W.: The stationary gravitational field near spatial infinity. Gen. Rel. Grav. (to be published)

9. Beig, R., Simon, W. : The multipole structure of the stationary gravitational field. (in preparation)

10. Hansen, R. : Multipole moments of stationary space-times. Math. Phys. 15, 46 (1974)

11. Hoenselaers, C. : Multipole moments of electrostatic space-times. Prog. Theor. Phys. 55, 406 (1976)

12. Hopf, E. : Uber den funktionalen, insbesondere analytischen Charakter der Lösungen elliptischer Differentialgleichungen zweiter Ordnung. Math. Z., 34, 194 (1931) 
13. Bers, L., John, F., Schechter, M. : Partial differential equations. New York: Wiley 1966, p. 118

14. Morrey, C. B. : On the analyticity of the solutions of analytic nonlinear elliptic systems of partial Differential equations. Am. J. Math. 80, 198 (1958)

15. Berger, M., Gauduchon, P., Mazet, E. : Le spectre d'une variété riemannienne. In: Lecture notes in mathematics, Vol. 194. Berlin, Heidelberg, New York: Springer 1971

16. Günther, P. : Spinorkalkül und Normalkoordinaten. Z. Angew. Math. Mech. 55, 205 (1975)

17. Penrose, R. : A spinor approach to general relativity. Ann. Phys. 10, 171 (1960)

Communicated by R. Geroch

Received May 6, 1980

Note added in proof: The stationary case has meanwhile been solved by the present authors. 